\title{
Protected Mammals of Nepal
}

- Bharat Gotame*

\section{Introduction}

The biological diversity of Nepal is of international importance due to its richness in fauna and flora owing to diverse topography to a wide altitudinal range and climatic zone. Nepal cover only $0.01 \%$ of land area of global scale but represent $4.5 \%$ of global mammalian species (suwal and verheght 1995), out of which a total of 181 mammal belongs to 12 orders and 39 families. Among them one mammal species, Himalayan Field Mouse (Apodemus Gurkha) is endemic to Nepal occurring in coniferous forest of central Nepal with 2200-3600m altitudinal range. Although these natural assets give Nepal a unique identity, numerous factors are engaged in the declination of these species. Other, three species namely Pigmy Hog (Sus Salvinus), Indian chevrotain (Moschiola Memina), Cheotah( Acinonyx jabatus) have become extinct from Nepal. Hispid hare (Caprologus hispidus), Nayan Sheep (Ovis ommon), Tibetain Antelope (Pantholops hodgsoni) is also believed to have vanished from Nepal as there is no recent information. Wild animal conservation, as a global issue, several organizations are devoting to face this issue. IUCN (International Union for conservation of nature and natural resources) is one of the independent international organizations established in 1948. IUCN has prepared Red list of threatened animals of world in 1986, 1988, 1990, 1994, where threatened animals are those which are known or suspected threatened with extinction and those known or believed to have become extinct all over the world. The red data book includes globally threatened animals on the basis of their population declination and range size of species. In 1994, IUCN Red list of threatened animals, 5929 species of animals out of total world species belonged to Nepal, which can be tabulated as follows:

\begin{tabular}{|llll|}
\hline S.N. & Species & World & Nepal \\
\hline 1 & Mammals & 741 & 28 \\
2 & Birds & 970 & 22 \\
3 & Amphibians & 169 & - \\
4 & Reptiles & 316 & 9 \\
5 & Fishes & 979 & - \\
6 & Invertebrates & 2754 & 2 \\
\hline
\end{tabular}

Nepal a state member of IUCN, Nepal red data book was published by IUCN- Nepal by categorizing the mammals of Nepal based on Red data book in 1994. Currently there are 58 mammals, 40 birds, 13 reptiles, 1 amphibian, 2 insects and 13 plants which appear in various categories.

\section{IUCN categories (developed in 1960 and revised in 1994)}

\section{Extinct (Ex)}

Species not definitely located in the wild during the past 50 years (criterion as used by CITES)

\section{Endangered (E)}

Taxa, danger of extinction and whose survival is unlikely if the casual factors continue operating.

Included are taxa whose numbers have been reduced to a critical level or whose habitats have been so drastically reduced that they are deemed to be in immediate

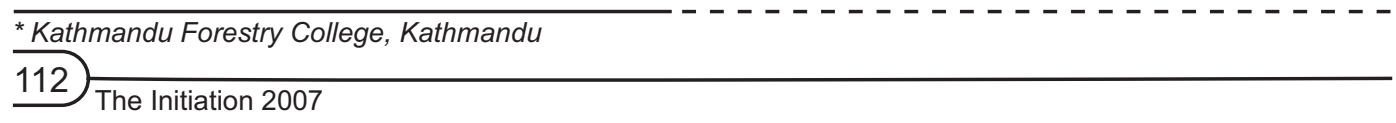


danger of extinction. Also included are taxa that may be extinct but have definitely been seen in the wild in the past 50 years.

\section{Vulnerable (V)}

Taxa likely to move into the endangered category in the near future if the casual factors continue operating. Included are taxa of which most or all the population is decreasing because of over-exploitation, extensive destruction of habitat or other environmental disturbances. Taxa with populations that have been seriously depleted and whose ultimate security has not yet been assured and taxa with population that is still abundant but is under threat from severe adverse factors throughout their range.

In practice, Endangered and vulnerable categories may include temporarily taxa whose populations are beginning to recover as a respect of remedial action. But whose recovery is insufficient to justify their transfer to another category.

\section{Rare (R)}

Taxa with small world populations that are not at present 'Endangered' or vulnerable but are at risk.

These taxa are usually localized within restricted geographical areas or habitat or are thinly scattered over a more extensive range.

\section{Indeterminate (I)}

Taxa known to be endangered, vulnerable or rare but where there is not enough information to say which of the three categories is appropriate.

\section{Insufficiently known (K)}

Taxa that are suspected but not definitely known to belong to any of the above categories, because of lack of information.

\section{Threatened (T)}

A general term used to denote species which are endangered, vulnerable, rare, indeterminate or insufficiently known and should not be confused with the use of same term by the US office of endangered species. In previous volumes it has been used to identify taxa comprised of several sub-taxa which have differing status categories.

\section{Commercially threatened (CT)}

Taxa not currently threatened with extinction but most or all of whose populations are threatened as a sustainable commercial resources or will become so unless their exploitation is regulated.

This category applies only to taxa whose populations are assumed to be relatively large. In practice, this category has only been used for marine species of commercial importance that are being over fished in several parts of their range.

In 1994 IUCN Red list of threatened animals, 5929 species of animals out of total world animal species, 61 species belong to Nepal which can be tabulated. 
Convention on International trade of Endangered species of Wild flora and fauna, established in 1973, and came into force in 1 July 1975, Nepal a state member of CITES in 1975 also follow its criteria as described below with 3 appendices. The main objective of CITES is to protect certain species of wild flora and fauna against over-exploitation through international trade.

\section{Appendix 1}

Contain species that are deemed to be threatened with extinction and which are or may be affected by trade. For these species trade is strictly regulated and only permitted in special circumstance. Appendix 1 include some highly threatened species, such as tiger, rhino, giant panda, Madagascar lemur, some crocodiles some orchids and some cacti.

\section{Appendix 2}

Contain species that although are not necessarily threatened, could become so if their trade is not properly controlled. Appendix 2 also contains some species that look so similar to species already listed that their trade is monitored and regulated in order to make control easier. Some of the species listed in Appendix 2 include- parrots, wild cats, some butterflies and stony corals.

Trade in Appendix 2 species is only permitted if the exporting country issues an export permit, while in the case of appendix 1 species where extra safeguards are necessary, an important permit is also required.

\section{Appendix 3}

Include species that are identified by any party as being subjected to regulations in that country and which require international cooperation to control trade.

\section{About the table}

1. Status of table

Accorded by global designation (IUCN) threat category and CITES Appendices) and National protected status (Nepal government- National park and wildlife reserve Act 1973) under this and next reading a dash indicates that no threat category has be ascribed.

2. Nepal Red data book status

Threat category ascribed for the species population within Nepal after suwal et al (1995)

3. Common name

After Carbet (1978) and (Corbet and Hill 1992)

\section{Abbreviation Used}

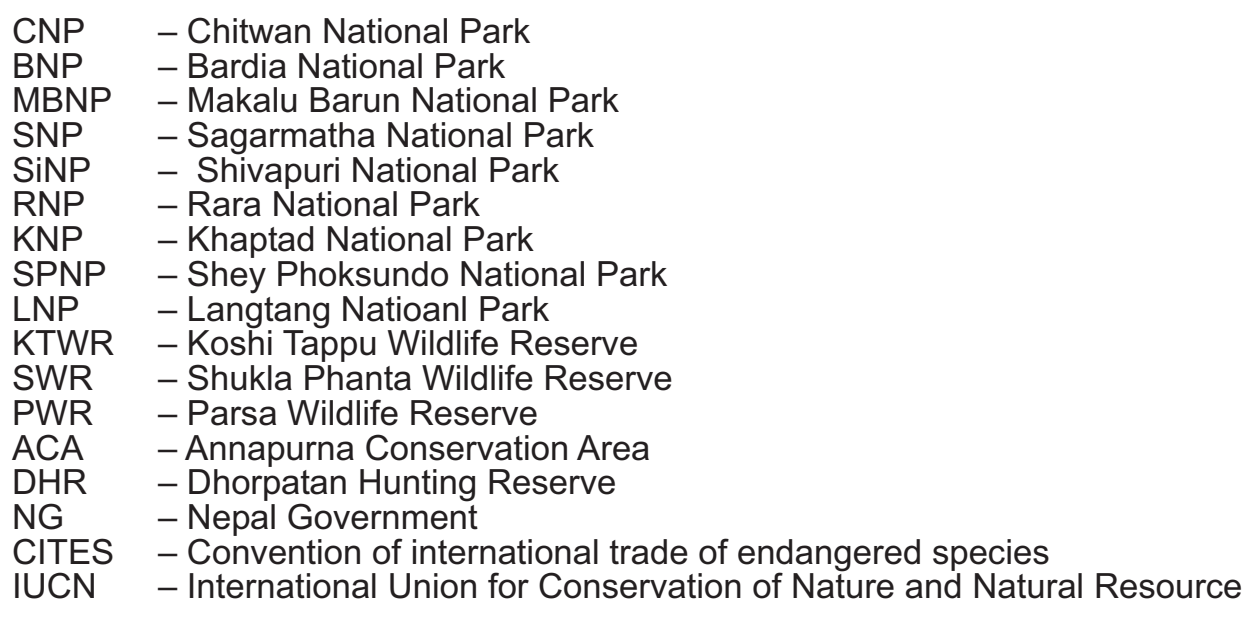




\begin{tabular}{|c|c|c|c|c|c|c|c|c|c|c|c|c|}
\hline 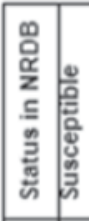 & . & 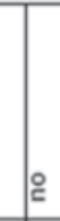 & 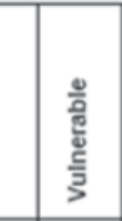 & 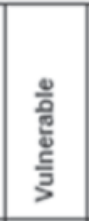 & 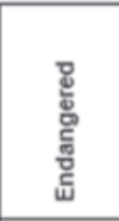 & n. & 高 & 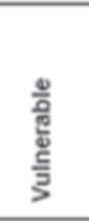 & 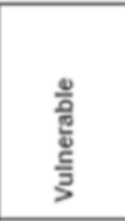 & 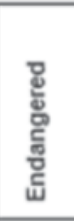 & 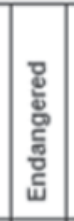 & 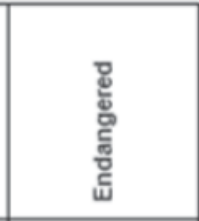 \\
\hline 㮣 & 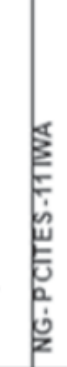 & 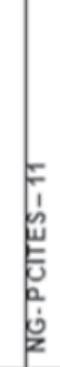 & 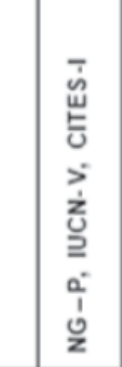 & 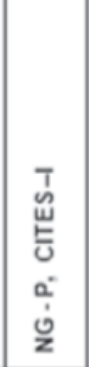 & 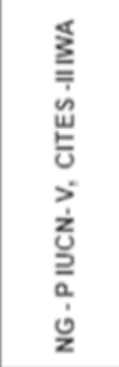 & 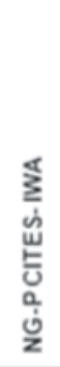 & $\mid \begin{array}{l}\mid \\
a \\
\vdots \\
2\end{array}$ & 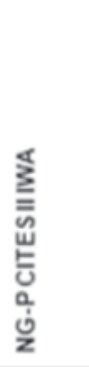 & 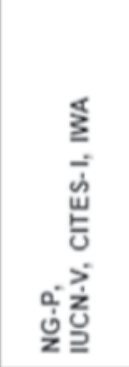 & 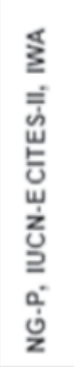 & 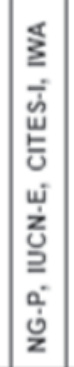 & 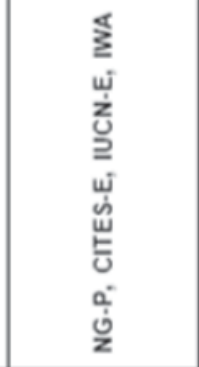 \\
\hline 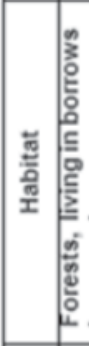 & & & 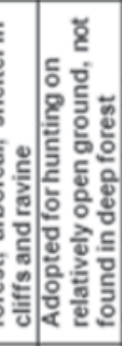 & 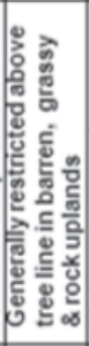 & 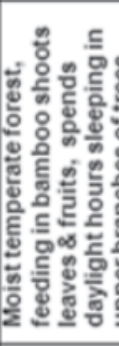 & & & 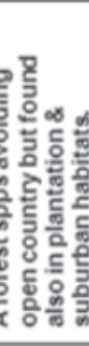 & 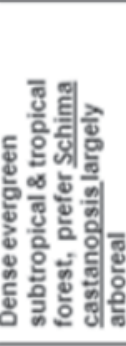 & 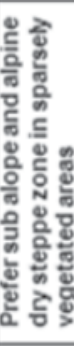 & 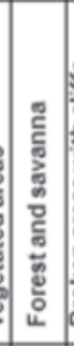 & 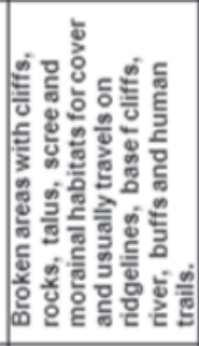 \\
\hline 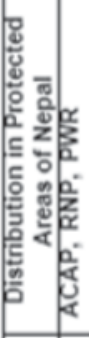 & 这 & 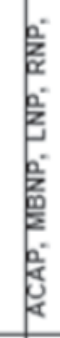 & 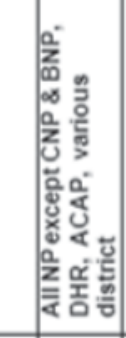 & 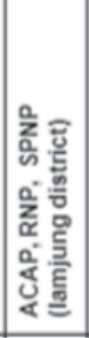 & 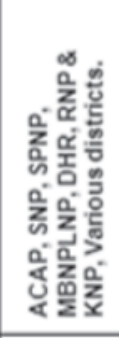 & 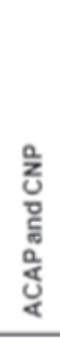 & 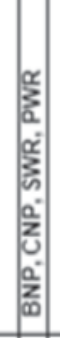 & 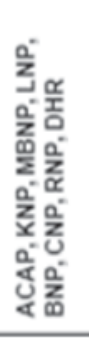 & 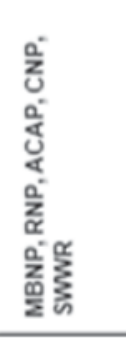 & 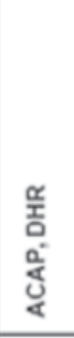 & 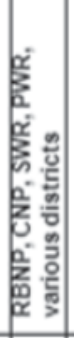 & 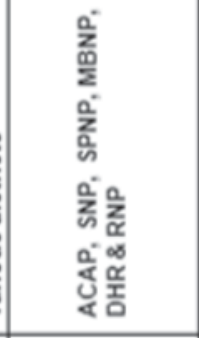 \\
\hline 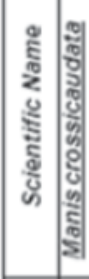 & : & 慈 & ㅎํำ & 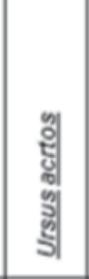 & 哀 & 형 & ㅎํㅊ & ㅎํำ & 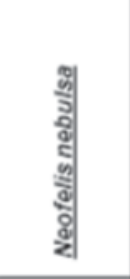 & 춘 & 형 & $\begin{array}{l}\text { : } \\
\text { : } \\
\text { : }\end{array}$ \\
\hline 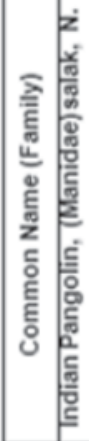 & 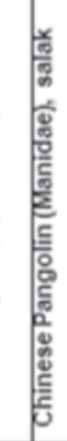 & 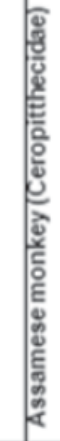 & 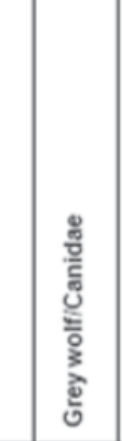 & 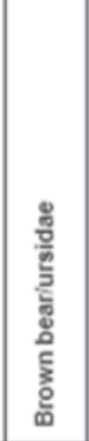 & 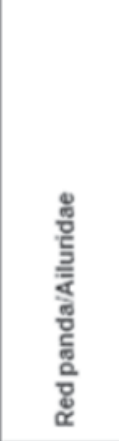 & 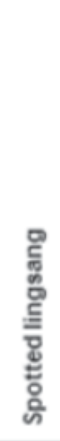 & : & 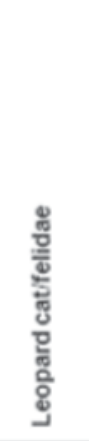 & 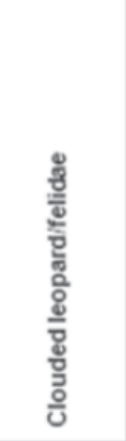 & 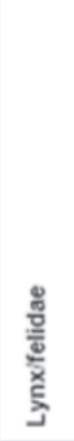 & 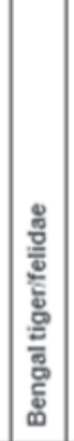 & 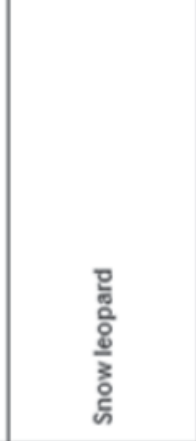 \\
\hline
\end{tabular}




\begin{tabular}{|c|c|c|c|c|c|c|c|c|c|c|c|c|}
\hline إن & 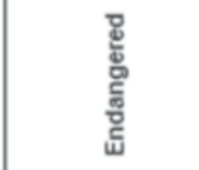 & 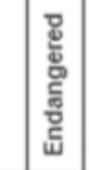 & ב & 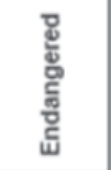 & & 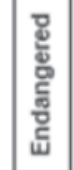 & 0 & , & 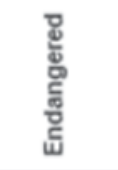 & 0 & 0 & a. \\
\hline 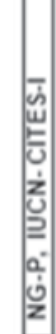 & 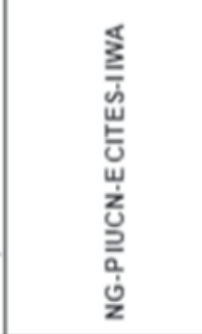 & 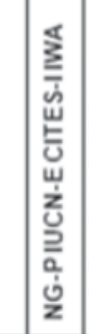 & 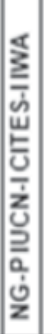 & 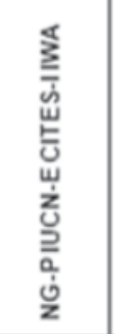 & 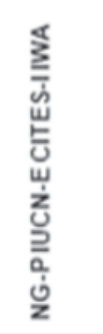 & 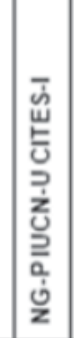 & 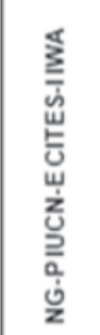 & 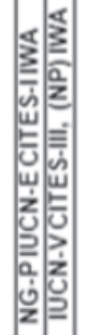 & 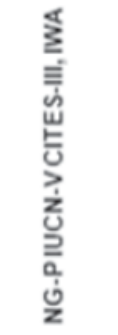 & 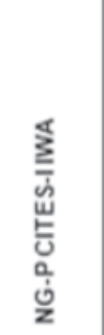 & 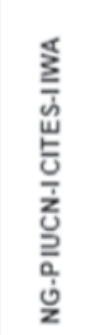 & 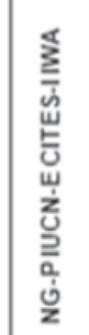 \\
\hline 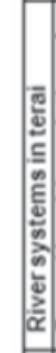 & 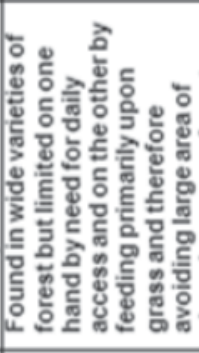 & 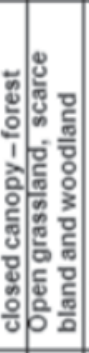 & 这 & 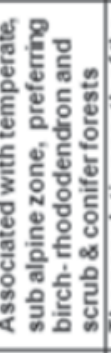 & 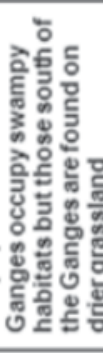 & 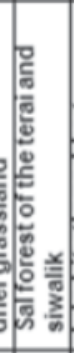 & 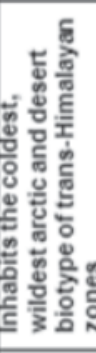 & 资 & 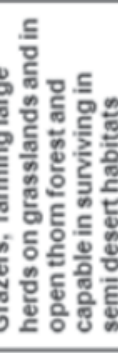 & 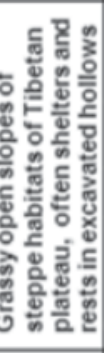 & 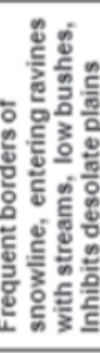 & 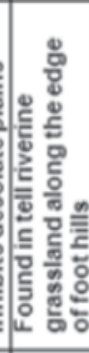 \\
\hline 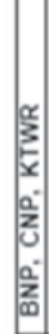 & 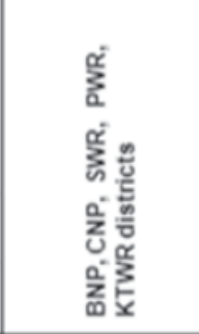 & 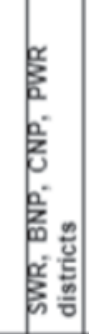 & & 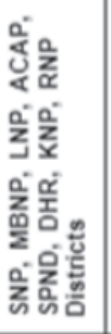 & 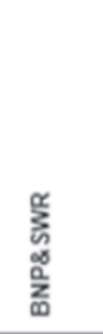 & 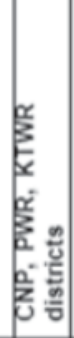 & 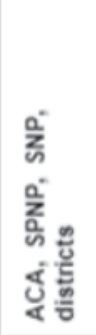 & 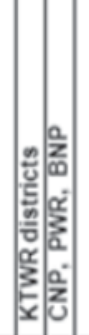 & $\sum_{\varpi}^{0}$ & 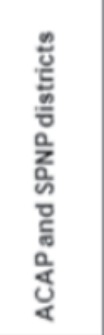 & 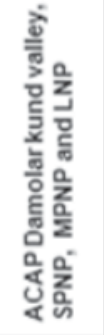 & 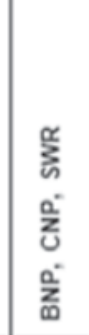 \\
\hline 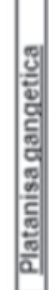 & 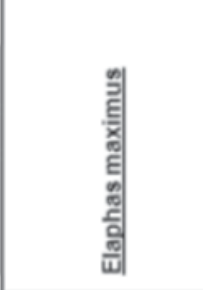 & 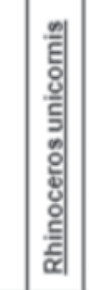 & & 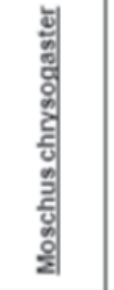 & $\begin{array}{l}\text { : } \\
\text { : }\end{array}$ & 형 & 氮 & 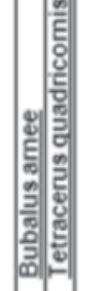 & 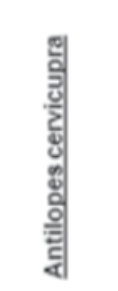 & $\begin{array}{l}\text { 혐 } \\
\text { 혛 } \\
\text { 혛 } \\
\text { 혛 }\end{array}$ & $\begin{array}{l}\text { 형 } \\
\text { 형 } \\
\text { 홓 }\end{array}$ & 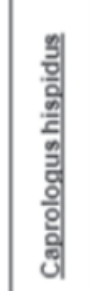 \\
\hline 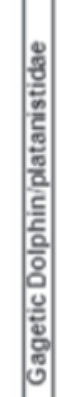 & 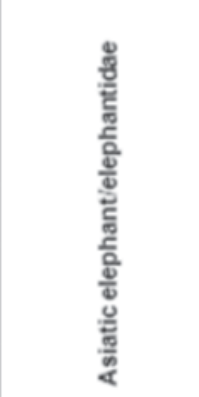 & 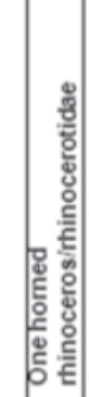 & & 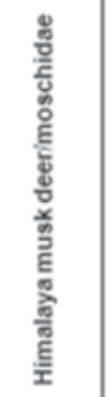 & 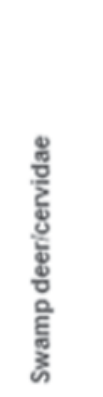 & 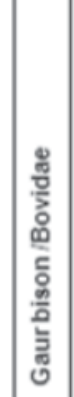 & 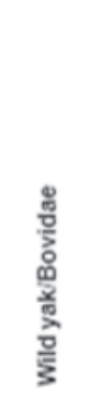 & 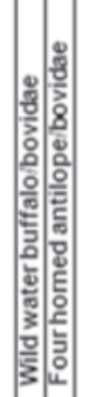 & 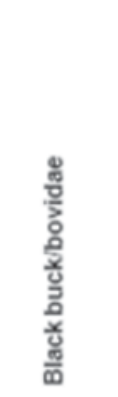 & 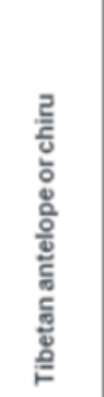 & 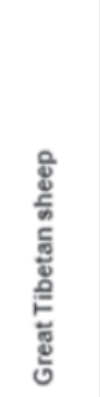 & 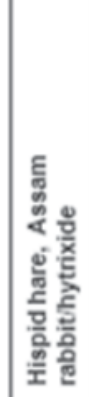 \\
\hline
\end{tabular}

